OPEN ACCESS

Edited by:

Peter E. Larsen,

Argonne National Laboratory (DOE),

United States

Reviewed by:

Michael Kevin Watters,

Valparaiso University, United States

Anna-Irini Koukkou,

University of loannina, Greece

${ }^{*}$ Correspondence:

Tingting Ning

ttning@whu.edu.cn

Specialty section:

This article was submitted to

Systems Microbiology,

a section of the journal

Frontiers in Microbiology

Received: 15 March 2017

Accepted: 09 August 2017

Published: 25 August 2017

Citation:

Ning $T$, Gong $X$, Xie $L$ and $M a B$

(2017) Gut Microbiota Analysis in Rats with Methamphetamine-Induced

Conditioned Place Preference.

Front. Microbiol. 8:1620.

doi: 10.3389/fmicb.2017.01620

\section{Gut Microbiota Analysis in Rats with Methamphetamine-Induced Conditioned Place Preference}

\author{
Tingting Ning ${ }^{1 *}$, Xiaokang Gong ${ }^{2}$, Lingling $\mathrm{Xie}^{3}$ and Baomiao $\mathrm{Ma}^{2}$ \\ ${ }^{1}$ College of Life Sciences, Jianghan University, Wuhan, China, ${ }^{2}$ Wuhan Institute of Biomedical Science, Jianghan University, \\ Wuhan, China, ${ }^{3}$ Key Laboratory of Combinatorial Biosynthesis and Drug Discovery (Wuhan University), Ministry of \\ Education, Wuhan University School of Pharmaceutical Sciences, Wuhan, China
}

Methamphetamine abuse is a major public health crisis. Because accumulating evidence supports the hypothesis that the gut microbiota plays an important role in central nervous system (CNS) function, and research on the roles of the microbiome in CNS disorders holds conceivable promise for developing novel therapeutic avenues for treating CNS disorders, we sought to determine whether administration of methamphetamine leads to alterations in the intestinal microbiota. In this study, the gut microbiota profiles of rats with methamphetamine-induced conditioned place preference (CPP) were analyzed through 16S rRNA gene sequencing. The fecal microbial diversity was slightly higher in the METH CPP group. The propionateproducing genus Phascolarctobacterium was attenuated in the METH CPP group, and the family Ruminococcaceae was elevated in the METH CPP group. Short chain fatty acid analysis revealed that the concentrations of propionate were decreased in the fecal matter of METH-administered rats. These findings provide direct evidence that administration of METH causes gut dysbiosis, enable a better understanding of the function of gut microbiota in the process of drug abuse, and provide a new paradigm for addiction treatment.

Keywords: methamphetamine, gut microbiota, 16S rRNA gene sequencing, short chain fatty acids, propionates

\section{INTRODUCTION}

Mammals including human beings live in a co-evolutionary association with a huge ecosystem of microorganisms living in the digest tract, mouth, skin, nose and vagina, which are termed the microbiota (Wu et al., 2015). The adult gastrointestinal tract contains a population of $10^{14}$ microorganisms, a number approximately 10 times the number of human cells in the body, and the collective genome of the microbiota amounts up to 150-fold more genes than are that of the human genome (Principi and Esposito, 2016). Although advances in the understanding of the role of microbiota in other areas of human health have yielded intriguing results (Moran and Shanahan, 2014; Rai et al., 2015; Biagi et al., 2016; Miyoshi and Chang, 2016), microbes and the brain have rarely been thought to interact. However, that belief is slowly changing (Luna and Foster, 2015; Parashar and Udayabanu, 2016; Principi and Esposito, 2016; Sarkar et al., 2016).

Recent investigations have rapidly uncovered a series of compelling connections between the gut and the brain (Diaz Heijtz et al., 2011; Erny et al., 2015; Luna and Foster, 2015; Ridaura and Belkaid, 2015; Parashar and Udayabanu, 2016). The relationship between the brain and the gut is primarily 
regulated at different levels including immune, neural, and endocrine levels (Kelly et al., 2015). The gut-brain-axis comprises the biochemical signaling that occurs between the gastrointestinal tract and the central nervous system (CNS). Indeed, microbiotic abnormalities have been associated with CNS disorders such as autism (Li and Zhou, 2016), Parkinson's disease (Scheperjans et al., 2015), schizophrenia (Severance et al., 2016), cognitive deficits (Frohlich et al., 2016), anxiety (Neufeld et al., 2011; Luna and Foster, 2015; Tarr et al., 2015), depression (Desbonnet et al., 2010; Luna and Foster, 2015; Macedo et al., 2016) and decrease in neurotransmitter levels (e.g., serotonin) (Yano et al., 2015).

Drug addiction, defined as "compulsive, out-of-control drug use despite negative consequences"1, remains one of the major public health issues worldwide; therefore, novel approaches are needed to be used as supplemental therapy or replace current behavioral and pharmacological therapy to treat addictive disorders (Cami and Farre, 2003). The emerging links between the gut microbiota and the CNS disorders have brought about a paradigm shift in neuroscience, with possible implications not only for understanding the pathophysiology but also treatment of many kinds of psychiatric disorders (Tillisch et al., 2013; AitBelgnaoui et al., 2014; Sarkar et al., 2016). Addiction may be viewed as a form of drug-induced neural plasticity. Therefore, there is a hypothesis raised now, that gut dysbiosis plays a key role during the development of drug addiction (Skosnik and Cortes-Briones, 2016).

Several possibilities exist wherein perturbations of gut microbiota might contribute to addiction. For example, the dietary and nutrition statuses of drug abusers are different from those of the general population and may in turn affect the composition of the gut microbiota. Volpe et al. (2014) have compared the composition of the intestinal flora between cocaine users and non-cocaine users and have found that cocaine users have a higher mean relative abundance of Bacteroidetes and a lower abundance of Firmicutes than non-users; are more likely to smoke; have a lower mean percentage of body fat; and consume more alcohol than non-users. In addition, stress is a crucial risk factor for the development of addiction to several kinds of drugs, including methamphetamine (Sinha and Jastreboff, 2013), and depression and anxiety are often comorbid with addiction. Growing evidence from studies in animals and humans indicates that gut dysbiosis can lead to altered stress responses (AitBelgnaoui et al., 2014; Kelly et al., 2015; Tarr et al., 2015; Leclercq et al., 2016; Rea et al., 2016; Sudo, 2016) and depressive symptoms, each of which can contribute to addiction (Jiang et al., 2015; Luna and Foster, 2015; Kunugi, 2016; Macedo et al., 2016). The most intriguing result is from a treatment perspective that the modification of gut bacterial profiles can reverse some of the above-mentioned abnormalities (Desbonnet et al., 2010; AitBelgnaoui et al., 2014; Tarr et al., 2015). More direct evidence has come from the observation that mice with fewer gut bacteria as a result of treatment with non-absorbable antibiotics show "an enhanced sensitivity to cocaine reward and an enhanced sensitivity to the locomotor sensitizing effects of repeated cocaine administration" (Kiraly et al., 2016).

${ }^{1}$ https://en.wikipedia.org/wiki/Substance_dependence
Methamphetamine ( $\mathrm{N}$-methyl-O-phenylisopropylamine; METH) is a commonly abused and highly addictive illicit drug. Regular use of METH is associated with a myriad of serious medical, cognitive, and psychosocial problems for drug users, which occur not only during abuse of the drug but also after cessation of use. The efficacy of the existing therapies for METH addiction, including cognitive therapy and substitution therapy, are still debated. Hence, novel approaches are needed to augment or replace current behavioral and pharmacological interventions for METH addictive disorders. The influence of the gut microbiome on the core symptoms of neuropsychiatric disorders is becoming increasingly recognized, and the microbiome might be a good target for novel treatment method. Therefore, the use of psychobiotics may provide a new way for treatments for drug addiction, but first, systematic studies are needed to examine whether administration of METH causes gut dysbiosis and whether there is a definite relationship between specific taxa and the use of METH.

In this study, a conditioned place preference (CPP) rat model was adopted, and high-throughput sequencing of 16S rRNA and bioinformatics analysis were performed to analyze the variations in gut microbiota in METH-administered rats compared with the control rats. The diversity of the bacterial community, the microbiota composition and the taxa that best characterized each group were analyzed. In addition, the concentrations of propionate and butyric acid in each group were analyzed.

\section{MATERIALS AND METHODS}

\section{Animals}

Male Sprague-Dawley rats (weighing $250 \pm 20$ g upon arrival; total $n=16)$ were housed individually under controlled temperature $\left(23 \pm 2^{\circ} \mathrm{C}\right)$ and humidity $(50 \pm 5 \%)$ and maintained on a $12 \mathrm{~h} / 12 \mathrm{~h}$ light/dark cycle with free access to food and water. All animal procedures were performed in accordance with the National Institutes of Health Guide for the Care and Use of Laboratory Animals, and the experiments were approved by Jianghan University Animal Use Committee.

\section{Chemicals and Drugs}

METH (98\%) was obtained from the Hubei Public Security Bureau and was dissolved in $0.9 \% \mathrm{NaCl}$. Propionate and butyric acid were purchased from Macklin Biochemical Technology Co., Ltd. (Shanghai, China).

\section{Conditioned Place Preference}

METH-induced CPP was performed as previously described, with minor modifications (Xue et al., 2012). The CPP system (Ningbo Anilab Software \& Instruments Co. Ltd., Zhejiang, China) was computer-controlled and consisted of three PVC compartments. Briefly, the CPP schedule consisted of three phases: preconditioning, conditioning, and post-conditioning. The sixteen rats were randomly assigned into two groups: the control $(n=8)$ and METH CPP group $(n=8)$. The preconditioning phase determined the baseline preference. Rats were initially placed in the middle chamber with the doors 


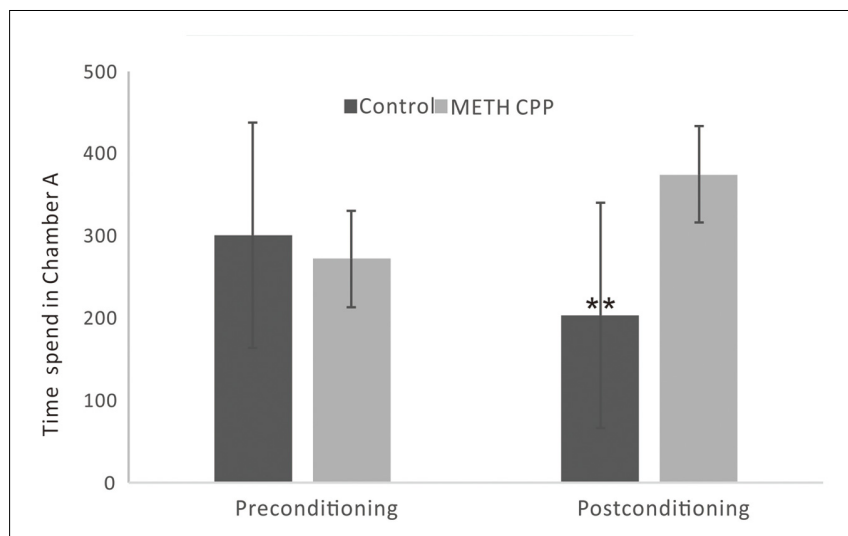

FIGURE 1 | Development of CPP induced by repeated administration of METH. Data are the mean \pm SEM of time spent in the METH-paired chamber (Chamber A) during the CPP tests. Double asterisk indicates significant difference between the METH CPP group and the control group $P<0.01$, $n=8$ rats per group.

removed for $15 \mathrm{~min}$. During the conditioning phase, each rat was treated for 14 days with alternate injections of either METH (1 $\mathrm{mg} / \mathrm{kg}$, i.p.) or saline $(2 \mathrm{~mL} / \mathrm{kg}$, i.p. $)$; thus, rats were treated with METH on day $1,3,5, \ldots, 13$, and were treated with saline on day $2,4,6, \ldots, 14$. Rats were confined to the white compartment for $45 \mathrm{~min}$ immediately after $\mathrm{METH}$ administration and to the black compartment after saline injection. In the postconditioning phase, rats were re-tested for METH-CPP by being allowed free access to both the white and black chambers for $15 \mathrm{~min}$. Rats in the control group received saline injections throughout the conditioning and post-conditioning phases. The time spent in each of the two compartments was automatically recorded for $15 \mathrm{~min}$ with a camera connected via electrical interface to a computer. Student's $t$-tests were used to determine whether significant place preference was established $(p<0.01$ was considered as a significant difference).

\section{Fecal Sample Collection and DNA Extraction}

Fecal sample collection was performed in metabolism cages. After the place preference test, animals were placed in the metabolism cages. The collection cup was sterilized before collection. Fecal samples were placed on ice as soon as possible and stored at $-80^{\circ} \mathrm{C}$. Fecal microbial DNA was extracted from $200 \mathrm{mg}$ of feces with a QIAamp DNA Stool Mini Kit (Qiagen, Hilden, Germany) according to the manufacturer's instructions. DNA was quantified using a NanoDrop ND-1000 spectrophotometer (Thermo Electron); integrity and size were assessed by $1.0 \%$ agarose gel electrophoresis. DNA was stored at $-20^{\circ} \mathrm{C}$ before analysis.

\section{High-Throughput Sequencing}

The bacterial communities in the fecal samples were investigated by Illumina MiSeq high-throughput sequencing.

The V3 and V4 regions of the $16 \mathrm{~S}$ rDNA gene were selected for PCR. The primers were barcoded-338F
$\left(5^{\prime}\right.$-ACTCCTACGGGAGGCAGCA- $\left.3^{\prime}\right)$ and 806R $\left(5^{\prime}-\right.$ GGACTACHVGGGTWTCTAAT- ${ }^{\prime} ; \mathrm{H}, \mathrm{W}$, and $\mathrm{V}$ were degenerate bases; $\mathrm{H}$ represented $\mathrm{A}$, $\mathrm{T}$ or $\mathrm{C} ; \mathrm{V}$ represented $\mathrm{G}$, A or $\mathrm{C}$; W represented $\mathrm{A}$ or $\mathrm{T}$ ), where the barcode was an eight-base sequence unique to each sample. The $20-\mu \mathrm{L}$ PCR reaction mixture was composed of $4 \mu \mathrm{L}$ of $5 \times$ FastPfu buffer, $2 \mu \mathrm{L}$ of $2.5 \mathrm{mM}$ dNTPs, $5 \mu \mathrm{M}$ each of forward and reverse primers, $0.4 \mu \mathrm{L}$ TransStart Fastpfu DNA Polymerase (TransGen Biotech, Beijing, China), and 10 ng DNA template. The following cycling parameters were used: maintain at $95^{\circ} \mathrm{C}$ for $2 \mathrm{~min}, 25$ cycles $\left(95^{\circ} \mathrm{C}\right.$ for $30 \mathrm{~s}, 55^{\circ} \mathrm{C}$ for $30 \mathrm{~s}$, and $72^{\circ} \mathrm{C}$ for $\left.30 \mathrm{~s}\right)$, and a final extension at $72^{\circ} \mathrm{C}$ for $5 \mathrm{~min}$. Triplicate reaction mixtures were pooled per sample, purified using an AxyPrep DNA gel extraction kit (Axygen, Union City, CA, United States) and quantified using a QuantiFluor-ST Fluorescence quantitative system (Promega, Madison, WI, United States). Amplicons from different samples were sent out for pyrosequencing on an Illumina MiSeq platform at Shanghai Majorbio Bio-Pharm Technology Co., Ltd. (Shanghai, China). All sequences have been deposited in the GenBank Short Read Archive (SRP093459).

\section{Processing of Sequencing Data}

Raw fastq files were demultiplexed and quality-filtered using QIIME (version 1.9.1) with the three criteria as mentioned in the reference (Song et al., 2016). Operational Taxonomic Units (OTUs) were clustered with a 97\% similarity cutoff using UPARSE (version $7.1^{2}$ ), and chimeric sequences were identified and removed using UCHIME. The taxonomy of each 16S rRNA gene sequence was analyzed with the RDP Classifier ${ }^{3}$ against the silva (SSU123)16S rRNA database by using a confidence threshold of $70 \%$.

\section{Statistical Analysis}

Community estimators were calculated and analyzed using Mothur version v.1.30.1 ${ }^{4}$, including richness estimators, the Ace index and Chao 1 index, and $\alpha$-diversity estimators, the Simpson index and Shannon index. The rarefaction curves were generated from the observed OTUs. Principal coordinate analysis (PCoA) was used to visualize clustering patterns between samples on the basis of $\beta$ diversity distances.

Microorganism features distinguishing fecal microbiota specific to METH addiction were identified using the Linear Discriminant Analysis (LDA) effect size (LEfSe) method $^{5}$ for biomarker discovery. A significance level of 0.05 and an effect-size threshold of 2 were used for all biomarkers.

\section{GC-MS Analysis of the Relative Concentration of Propionate and Butyric Acid in the Fecal Matter}

The extraction procedure of short chain fatty acids was performed as previously described (Hu et al., 2012). Briefly, a

\footnotetext{
${ }^{2}$ http://drive5.com/uparse/

${ }^{3}$ http://rdp.cme.msu.edu/

${ }^{4}$ http://www.mothur.org/wiki/Schloss_SOP\#Alpha_diversity

${ }^{5}$ http://huttenhower.sph.harvard.edu/lefse/
} 
TABLE 1 | Comparison of phylotype coverage and diversity estimation of the $16 \mathrm{~S}$ rRNA gene libraries for individuals at $97 \%$ similarity from the pyrosequencing analysis.

\begin{tabular}{|c|c|c|c|c|c|c|c|}
\hline \multirow[t]{2}{*}{ Sample ID } & \multirow[t]{2}{*}{ Reads } & \multicolumn{6}{|c|}{$97 \%$} \\
\hline & & OTU & Ace & Chao & Coverage & Shannon & Simpson \\
\hline C 1 & 19921 & 419 & 456 & 458 & 0.997139 & 4.46 & 0.0297 \\
\hline C 2 & 19921 & 316 & 380 & 375 & 0.996486 & 3.34 & 0.1144 \\
\hline C 3 & 19921 & 325 & 383 & 384 & 0.996536 & 3.6 & 0.0839 \\
\hline C 4 & 19921 & 266 & 315 & 334 & 0.997038 & 3.12 & 0.1565 \\
\hline C 5 & 19921 & 334 & 388 & 381 & 0.996737 & 3.41 & 0.1237 \\
\hline C 6 & 19921 & 271 & 343 & 350 & 0.996687 & 3.03 & 0.1948 \\
\hline C 7 & 19921 & 290 & 357 & 350 & 0.996587 & 3.37 & 0.1057 \\
\hline C 8 & 19921 & 319 & 388 & 396 & 0.996185 & 2.89 & 0.2055 \\
\hline METH CPP 1 & 19921 & 363 & 404 & 418 & 0.996938 & 3.83 & 0.0896 \\
\hline METH CPP 2 & 19921 & 347 & 402 & 390 & 0.996687 & 4.05 & 0.0414 \\
\hline METH CPP 3 & 19921 & 375 & 418 & 411 & 0.996988 & 4 & 0.0505 \\
\hline METH CPP 4 & 19921 & 304 & 362 & 367 & 0.996687 & 3.42 & 0.1071 \\
\hline METH CPP 5 & 19921 & 362 & 414 & 424 & 0.996436 & 3.92 & 0.0491 \\
\hline METH CPP 6 & 19921 & 363 & 436 & 435 & 0.995884 & 3.48 & 0.1465 \\
\hline METH CPP 7 & 19921 & 438 & 490 & 492 & 0.996436 & 4.61 & 0.0228 \\
\hline METH CPP 8 & 19921 & 292 & 364 & 364 & 0.996687 & 3.92 & 0.0475 \\
\hline
\end{tabular}

400-mg stool sample was used to extract short chain fatty acids using $2 \mathrm{~mL}$ water. The short chain fatty acids extracted by water were lyophilized and then estered by adding $500 \mu \mathrm{L} 5 \%$ sulphuric acid/butanol at $90^{\circ} \mathrm{C}$ for $2 \mathrm{~h}$. Then, the esterification was stopped by addition of $500 \mu \mathrm{L} 0.9 \% \mathrm{NaCl}$. Derivatives of short chain fatty acids were then extracted with $500 \mu \mathrm{L} n$-hexane and analyzed with GC-MS.

GC-MS analysis of fatty acid esters dissolved in hexane phase was performed and quantified using a TRACE Ultra gas chromatograph connected to a TSQ Quantum XLS triple quadrupole mass spectrometer (GC-MS, Thermo Scientific Inc., San Jose, CA, United States). The hexane phase was analyzed on a TRACE TR-5MS $(30 \mathrm{~m} \times 0.25 \mathrm{~mm} \times 0.25 \mu \mathrm{m})$, after a $1-\mu \mathrm{L}$ injection with an auto-sampler (AI/AS 3000). Injections were performed in split mode with a split ratio of 20:1. Helium was used as the carrier gas, with a flow rate of $1 \mathrm{~mL} / \mathrm{min}$. The inject inlet and ion source temperatures were 220 and $240^{\circ} \mathrm{C}$, respectively. The temperature sequence was programmed as follows: $60^{\circ} \mathrm{C}$ as an initial temperature for $6 \mathrm{~min}$, and then a $4^{\circ} \mathrm{C} / \mathrm{min}$ ramp to $120^{\circ} \mathrm{C}$, held at $120^{\circ} \mathrm{C}$ for $10 \mathrm{~min}$. Each sample was tested three times. Student's $t$-test was used to determine whether difference existed between the two groups $(p<0.05)$.

\section{RESULTS}

\section{METH-Induced CPP}

During the preconditioning stage of the CPP paradigm, there was no difference between the time spent in chamber A between the control group and the METH CPP group. After treatment with METH for 7 sessions, a significant CPP was established. Rats spent 374 s of their time in the METH-paired, naturally unpreferred white compartment. In contrast, rats in the control group showed a natural preference for the black compartment, and no CPP was seen (Figure 1).

\section{Diversity of the Microbial Community}

In total, approximately 318,736 sequence reads of $16 \mathrm{~S}$ rRNA genes with an average length of approximately 439 bp were obtained after trimming and chimera removal. A 97\% similarity cut-off was used to delineate OTUs in the downstream analyses. After subsampling, a total of 5384 OTUs were acquired (Table 1).
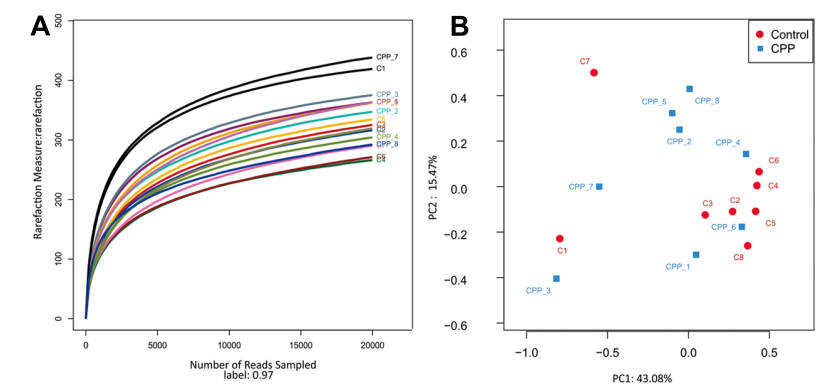

FIGURE 2 | Rarefaction curve of $16 S$ rDNA sequences of the 16 samples and principal coordinate analysis (PCOA) of the samples using

Unweighted-UniFrac from pyrosequencing. (A) Rarefaction curves based on the $16 \mathrm{~S}$ rRNA gene sequencing of the 16 samples from the METH CPP group and the control group ( $\mathrm{C}$ represents the control group, CPP represents the METH CPP group). The rarefaction curves suggested that the bacterial community was represented well because the curves became relatively flat as the number of sequences analyzed increased. (B) Principal coordinate analysis (PCOA) of the samples using Unweighted-UniFrac from

pyrosequencing. The red dots represent the control group, and the blue squares represent the METH-CPP group. The fecal microbiotas of the two groups could not be divided into clusters according to community composition using Unweighted UniFrac metrics and could not be separated clearly by PCoA analysis (ADONIS test, $R^{2}=0.08276, p$-value $=0.191$ ). 


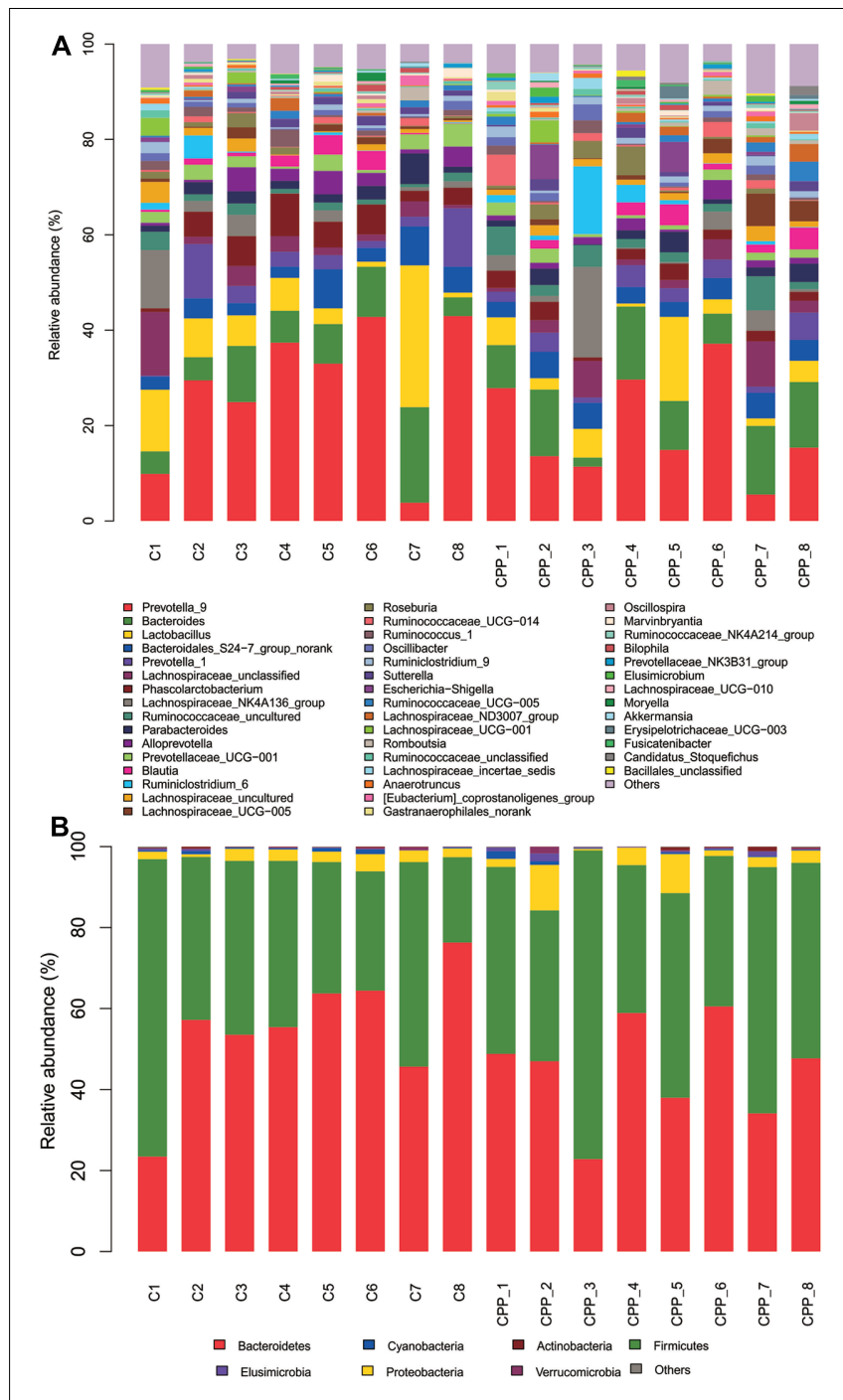

FIGURE 3 | Bacterial community structures in all samples at the genus level (A) and phylum level (B). The abundance is presented in terms of the percentage of the total effective bacterial sequences in the sample.

Rarefaction curves suggested that the bacterial community was well represented because the curves became relatively flat as the number of sequences analyzed increased (Figure 2). Species richness is the number of bacterial species assigned by OTUs detected in the samples. Richness estimates were obtained from the observed number of species by extrapolation using estimators such as the ACE and Chaol indices (Table 1). ACE and Chaol were estimated to be $376.25 / 378.5$ in the control group and 411.25/412.625 in the METH CPP group, respectively, but there were no differences between these two groups. However, the microbial diversity, indicated by the Shannon and Simpson indices, was different between the two groups. The Shannon and Simpson indices were 3.403/0.127 in the control group and 3.904/0.069 in the METH CPP group ( $p$-value for the Shannon and Simpson indices between the two groups was 0.035 and 0.038 , respectively, as determined by a two-tailed $t$-test). Because a higher Shannon and lower Simpson index indicate higher community diversity, the results suggest that the fecal microbial diversity in the METH group was somewhat greater than that in the control group. This result was unexpected, because a greater bacterial diversity is conceived to be beneficial to human health, but the role of bacterial diversity in CNS function remains to be explored.

Owing to the complexity of each sample, the fecal microbiotas of the two groups could not be divided into clusters according to community composition by using unweighted UniFrac metrics and could not be separated clearly by PCoA analysis (ADONIS test, $R^{2}=0.08276, p$-value $=0.191$; Figure 2).

\section{Altered Microbiota Composition in the METH CPP Group}

Bacteria from the 16 samples demonstrated similar richness but different abundances. The relative bacterial community abundance on the phylum and genus levels are shown in Figure 3. LEfSe was used to determine the taxa that best characterized each population. LEfSe scores measure the consistency of differences in the relative abundance between taxa in the groups analyzed with a higher score, thus indicating higher consistency. LDA revealed distinct taxa in the microbiome of the control group versus the METH CPP group (Figure 4). Ruminococcaceae, Bacillus, Cetobacterium, Fusobacteria, and Aeromonas, which were abundant in the METH CPP group, were the key phylotypes that contributed to the difference in the intestinal microbiota composition between the METH CPP and control group. In addition, Phascolarctobacterium, which was more abundant in the control group, was the representative phylotype in the normal group (Figure 5).

\section{Analysis of Propionate and Butyric Acid}

Because the genus Phascolarctobacterium has been characterized by producing propionate, and this genus was decreased in the METH CPP group, we proposed that propionate was also attenuated by METH administration. To test this hypothesis, we analyzed the relative concentration of propionate in the fecal matter of the two groups by GC-MS. The data showed that the relative concentration of propionate in the control group was more than twice that in the METH CPP group. We also observed a trend in the variation of butyric acid, but no differences were found between these two groups (Figure 6).

\section{DISCUSSION}

In this study, we found differences in a-diversity and in the relative abundance of specific bacterial taxa in gut microbiota and in the concentration of propionate associated with METH administration. To date, there has been only one study that has compared the fecal microbiota of cocaine users and non-users and found no significant group differences in microbiota diversity (Volpe et al., 2014). In animal models of drug dependence, in contrast to studies of clinical samples, variables, including diet, living environment, age, sex, etc., can be controlled for easily; thus, the influence by factors other than drug administration can 

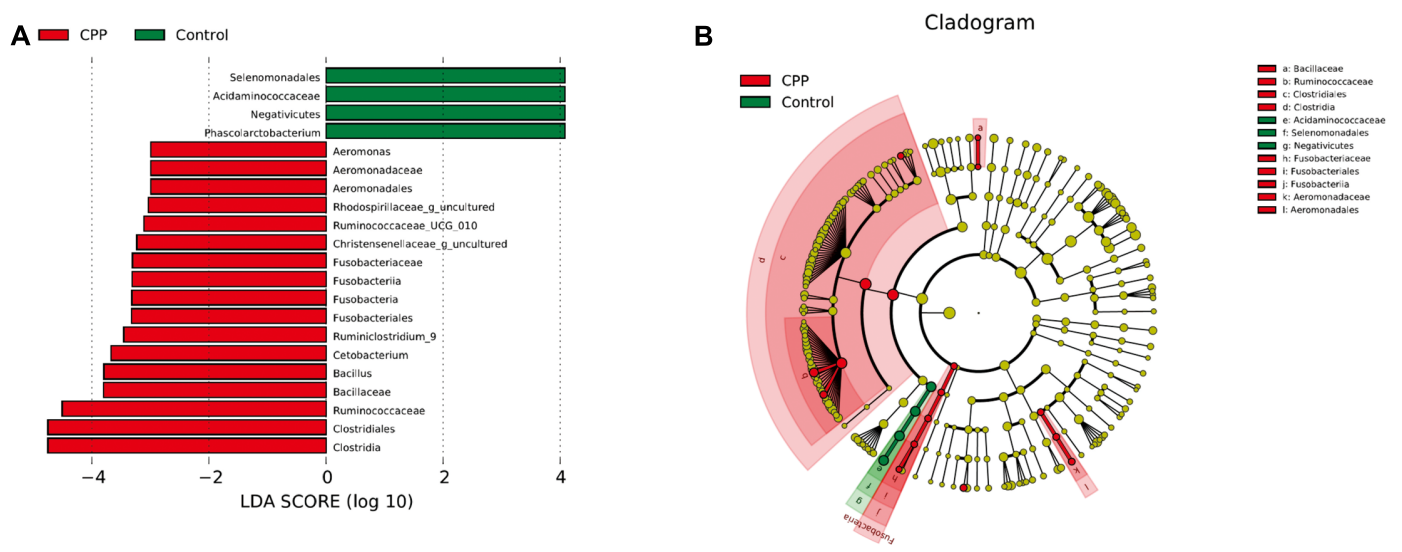

FIGURE 4 | Linear Discriminant Analysis (LDA) shows distinct gut microbiome composition in the METH CPP group and the control group. (A) LDA scores, as calculated by the LEfSe of taxa differentially abundant in the two groups. (B) LEfSe cladogram representing differentially abundant taxa. Only taxa with LDA scores of more than 2 are presented.
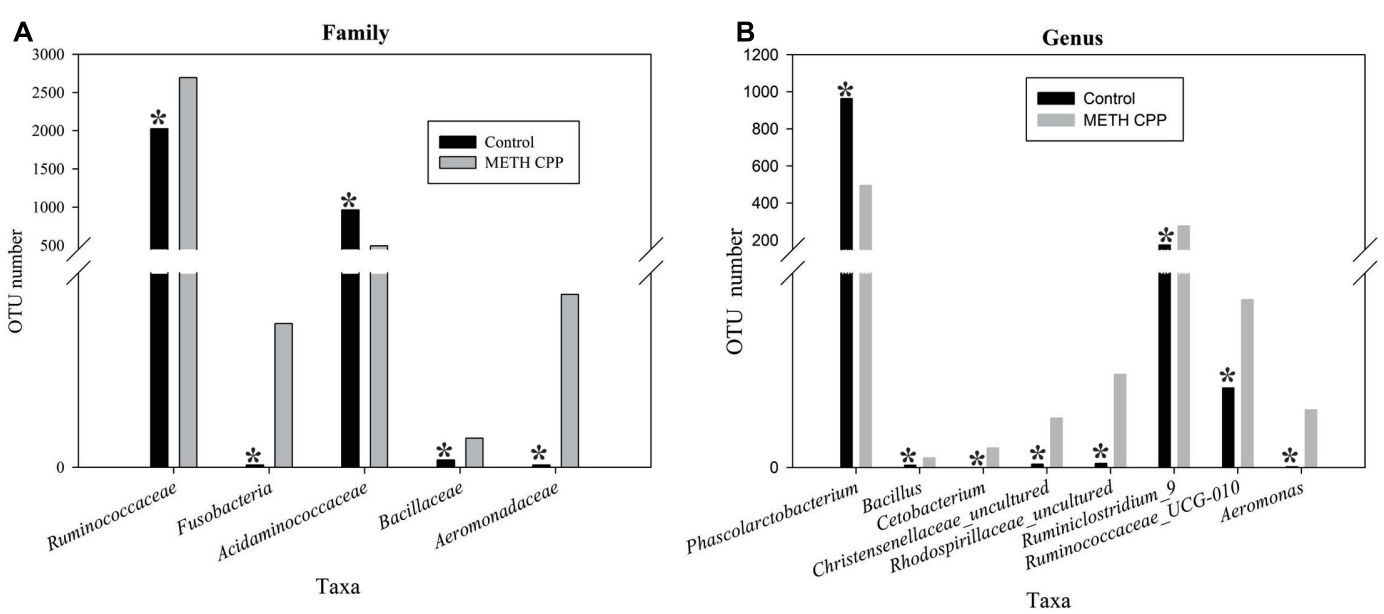

FIGURE 5 | Taxonomic differences of fecal microbiota between the control and METH CPP groups. Comparison of relative abundance at the bacterial family (A) and genus (B) levels between these two groups. Student's $t$-test was used to determine whether differences existed between the two groups. ${ }^{*} p<0.05$.

be minimized. This is the first study to explore changes in the fecal microbiota of a drug-administered animal model. In the present study, the fecal microbial diversity (estimated using the Shannon and Simpson indices) was greater in METH CPP rats. Although greater bacterial diversity is potentially beneficial to human health, its role in CNS function should be explored in more depth. A previous study has demonstrated greater gut microbe diversity in active major depressive disorder patients (Jiang et al., 2015). Furthermore, in the feces of autistic children increased gut microbe diversity and richness have also been detected (Finegold et al., 2010). Therefore, why METH administration could result in increased bacterial diversity remains to be clarified in the future.

In this study, gut dysbiosis was characterized by significant taxonomical differences between three of the major phyla in the METH CPP versus control subjects. Several taxa from Proteobacteria and Fusobacteria were significantly more abundant in METH CPP subjects, whereas the proportion of different families of Firmicutes showed different variation trends in these two groups. Acidaminococcaceae was more abundant in the control group, and Ruminococcaceae and Bacillaceae were elevated in the METH CPP group.

Firmicutes are the largest phylum of bacteria, comprising more than 200 genera, most of which belong to the gram-positive bacteria, in which lipoteichoic acid is a major constituent of the cell wall. The genus Phascolarctobacterium, belonging to the family Acidaminococcaceae, was the characteristic taxon in the control group. The relative abundance of this genus in the control group was approximately twofold more than in the METH CPP group (Figure 5). This genus has frequently been reported in relationship to obesity and nutrition and had significant positive correlations with the nutritional index in humans (Million et al., 2016) and a high-fat diet in rats (Lecomte et al., 2015). In a clinical study, the mean relative abundance of Firmicutes in cocaine users has been found to be lower than that in non-users (Volpe et al., 

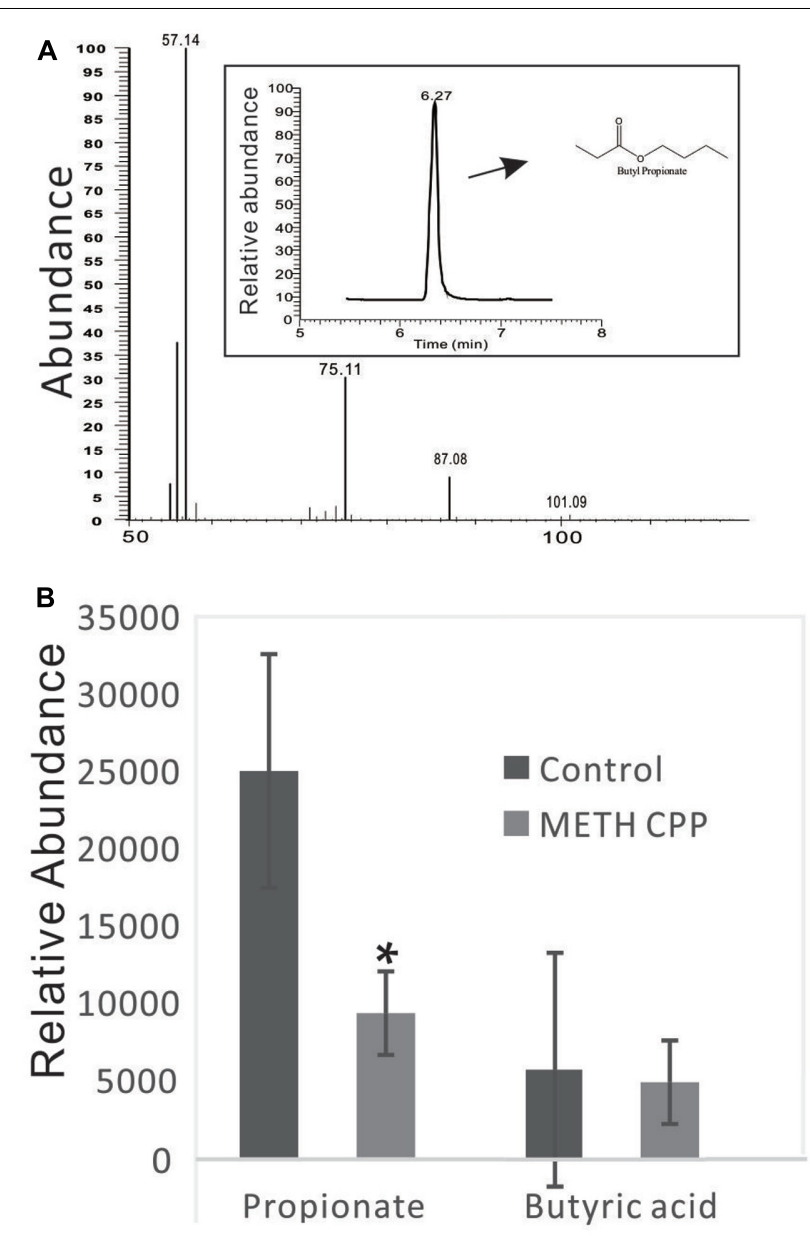

FIGURE 6 | Analysis of propionate and butyric acid in the fecal matter of the control group and the METH CPP group. (A) Representative GC-MS spectrum of the butanol derivative of propionate in the fecal sample. (B) Relative abundance of propionate and butyric acid in the fecal matter of the control and METH CPP group tested through GC-MS (Means \pm SEM). Each sample was analyzed three times. *Indicates difference between the control group and the METH CPP group $(p<0.05)$.

2014); this trend was the same as that shown in body fat and the healthy eating index. Inspired by the findings suggesting a role of Firmicutes in obesity, we compared the weights of these two groups, but no differences were observed. This genus has been characterized on the basis of its utilization of succinate and production of propionate (Watanabe et al., 2012). Propionate, a C3-short chain fatty acid, along with other short-chain fatty acids (SCFAs), small organic monocarboxylic acids with less than six carbon atoms, are major microbial metabolites produced during anaerobic fermentation in the gut (Stilling et al., 2016). SCFAs are the most important and pleiotropic functional components of microbe-to-host signaling (Fukumoto et al., 2003). They have shown promising effects in many kinds of diseases including neurological disorders (Yang et al., 2013; Koh et al., 2016; Stilling et al., 2016). It is now well established that SCFAs modulate colonic motility by stimulating serotonin secretion from gut enterochromaffin cells (Fukumoto et al., 2003; Yano et al., 2015).
A recent study has found that GF mice have immature and less active microglia, an effect that can be normalized by addition of an SCFA cocktail consisting of acetate, propionate and butyrate to the drinking water (Erny et al., 2015).

We presumed that the decrease in Phascolarctobacterium abundance in the METH CPP group would result in the attenuation of the concentration of propionate. To validate this hypothesis, we analyzed the relative concentration of propionate in the fecal matter of the two groups by GC-MS (Figure 6). As we expected, the concentration of propionate in the control group was more than twice that in the METH CPP group. Further studies should be carried out to validate whether serotonin release and the function of microglia are affected.

Propionate is produced in the human large intestine by microbial fermentation, and has potential health-promoting effects (Al-Lahham et al., 2010). Although, together with acetate and butyric acid, propionate was the major short chain fatty acid produced in the gut by colonic microbiota of fermentation dietary fibers, most previous studies have focused on butyrate and to a lesser extent acetate. Consequently, the potential effects of propionic acid on human health have been underestimated (Stilling et al., 2016). Propionate can readily cross both the gut-blood and blood-brain barriers, thus it could lead to neuroactive effects, but the manner in which propionate may influence the CNS is unknown. Very recently and interestingly, intraventricular infusions of PA have been demonstrated to cause behavioral and brain abnormalities in rats which were similar to those seen in humans with autism (MacFabe et al., 2007; Arora et al., 2011; MacFabe et al., 2011; Shultz et al., 2015). Our results thus shed new lights on the role of propionate in human health.

Two genera, Ruminococcaceae_UCS-010 and Ruminiclostridium_9, which were increased in the METH CPP group, belonged to the family Ruminococcaceae. This family displayed positive correlations with the butyrate/SCFAs ratio and has been reported to be affected by different kinds of mental diseases (Yang et al., 2013). However, we found no differences in the relative abundance of $n$-butyric acid between the two groups. Stressor exposure significantly changed the structure of the colonic mucosa-associated microbiota in control mice and resulted in anxiety-like behavior, but the effect was not evident in mice fed milk oligosaccharides. Several phyla were increased in control mice compared with prebiotic-fed mice, including unclassified Ruminococcaceae (Tarr et al., 2015). This result indicated that Ruminococcaceae correlate with anxiety, which is a key characteristic of METH abusers. Short-term exposure to high-energy diets impairs memory. Rats consuming saturated fatty acids and sugar, compared with controls and rats consuming polyunsaturated fatty acids, were impaired in hippocampal dependent place recognition memory, even though all rats consumed similar amounts of energy. Ruminococcaceae have shown a range of macronutrient-specific negative correlations with place memory (Beilharz et al., 2016); thus, this phylum plays an important role in cognitive function. In our results, the abundance of the genera Ruminiclostridium_9 and Ruminococcaceae_UCG_010, both of which belong to the family Ruminococcaceae, was much higher in the METH CPP group than in the control group. METH abuse may result 
in cognitive deficits, including increased anxiety, impaired memory. Therefore, the elevated abundance of Ruminococcaceae may be involved in the deleterious effects of METH on cognitive processes.

It would be more valuable to clarify the relationship between these alterations and METH addiction. Behavior study to confirm whether supplementation propionate in the diet could change the preference for the drug would be helpful. Using models like elevated plus-maze and Morris water maze that assess the anxiety and place memory of the METH-administrated rats could help to validate the function of Ruminococcaceae in METH dependence. In addition, transcription/immunohisto-chemical analysis of gene targets such as fatty acid receptor-FFAR3 will contribute to reveal the gut-brain circuit via which propionate lead to neuroactive effects (De Vadder et al., 2014).

In summary, recent research has provided strong evidence for the role of the commensal gut microbiota in brain function and behavior, but, to date, there has been no systematic study of the involvement of gut microbiota in drug addiction. This work is the first meaningful effort to determine the relationship between METH administration and gut microbiota. The fecal microbial diversity was greater in METH CPP rats than that in the control rats, and the phylum Firmicutes accounted for the majority of the altered taxa. The most significant finding is that the propionate-producing genus Phascolarctobacterium was repressed by METH administration, and this result was further confirmed by the decrease in propionate in the fecal matter. In

\section{REFERENCES}

Ait-Belgnaoui, A., Colom, A., Braniste, V., Ramalho, L., Marrot, A., Cartier, C., et al. (2014). Probiotic gut effect prevents the chronic psychological stressinduced brain activity abnormality in mice. Neurogastroenterol. Motil. 26, 510-520. doi: 10.1111/nmo.12295

Al-Lahham, S. H., Peppelenbosch, M. P., Roelofsen, H., Vonk, R. J., and Venema, K. (2010). Biological effects of propionic acid in humans; metabolism, potential applications and underlying mechanisms. Biochim. Biophys. Acta 1801, 1175-1183. doi: 10.1016/j.bbalip.2010.07.007

Arora, T., Sharma, R., and Frost, G. (2011). Propionate. Anti-obesity and satiety enhancing factor? Appetite 56, 511-515. doi: 10.1016/j.appet.2011.01.016

Beilharz, J. E., Kaakoush, N. O., Maniam, J., and Morris, M. J. (2016). The effect of short-term exposure to energy-matched diets enriched in fat or sugar on memory, gut microbiota and markers of brain inflammation and plasticity. Brain Behav. Immun. 57, 304-313. doi: 10.1016/j.bbi.2016.07.151

Biagi, E., Franceschi, C., Rampelli, S., Severgnini, M., Ostan, R., Turroni, S., et al. (2016). Gut microbiota and extreme longevity. Curr. Biol. 26, 1480-1485. doi: 10.1016/j.cub.2016.04.016

Cami, J., and Farre, M. (2003). Drug addiction. N. Engl. J. Med. 349, 975-986. doi: 10.1056/NEJMra023160

De Vadder, F., Kovatcheva-Datchary, P., Goncalves, D., Vinera, J., Zitoun, C., Duchampt, A., et al. (2014). Microbiota-generated metabolites promote metabolic benefits via gut-brain neural circuits. Cell 156, 84-96. doi: 10.1016/ j.cell.2013.12.016

Desbonnet, L., Garrett, L., Clarke, G., Kiely, B., Cryan, J. F., and Dinan, T. G. (2010). Effects of the probiotic Bifidobacterium infantis in the maternal separation model of depression. Neuroscience 170, 1179-1188. doi: 10.1016/j.neuroscience. 2010.08.005

Diaz Heijtz, R., Wang, S., Anuar, F., Qian, Y., Bjorkholm, B., Samuelsson, A., et al. (2011). Normal gut microbiota modulates brain development and behavior. Proc. Natl. Acad. Sci. U.S.A. 108, 3047-3052. doi: 10.1073/pnas.10105 29108 contrast, the family Ruminococcaceae, which has been reported to have a positive relationship with anxiety and a negative relationship with memory, was elevated in the METH CPP group. Although further clinical and in vivo studies are needed to better understand the mechanisms underlying the link between gut microbiota and drug administration, these findings suggest that gut microbiota manipulation might be a rational approach to treat drug addiction.

\section{AUTHOR CONTRIBUTIONS}

This study was conceived and designed by TN. The experiments were conducted by TN and XG. The data analysis was done by TN. LX did the GC-MS analysis of propionate in the fecal matter. BM did the DNA extraction work. All authors reviewed the manuscript.

\section{ACKNOWLEDGMENTS}

This study was supported by the project of the Education Department of Hubei Province (B2016300). We thank Professor Chaoying $\mathrm{Li}$ in the Wuhan Institute of Biomedical Science and Jianghan University for help in proof-reading the article. We thank Dongmei Huang in Majorbio Bio-Pharm Technology Co., Ltd., for help in data analysis.

Erny, D., Hrabe De Angelis, A. L., Jaitin, D., Wieghofer, P., Staszewski, O., David, E., et al. (2015). Host microbiota constantly control maturation and function of microglia in the CNS. Nat. Neurosci. 18, 965-977. doi: 10.1038/nn.4030

Finegold, S. M., Dowd, S. E., Gontcharova, V., Liu, C., Henley, K. E., Wolcott, R. D., et al. (2010). Pyrosequencing study of fecal microflora of autistic and control children. Anaerobe 16, 444-453. doi: 10.1016/j.anaerobe.2010.06.008

Frohlich, E. E., Farzi, A., Mayerhofer, R., Reichmann, F., Jacan, A., Wagner, B., et al. (2016). Cognitive impairment by antibiotic-induced gut dysbiosis: analysis of gut microbiota-brain communication. Brain Behav. Immun. 56, 140-155. doi: 10.1016/j.bbi.2016.02.020

Fukumoto, S., Tatewaki, M., Yamada, T., Fujimiya, M., Mantyh, C., Voss, M., et al. (2003). Short-chain fatty acids stimulate colonic transit via intraluminal 5-HT release in rats. Am. J. Physiol. Regul. Integr. Comp. Physiol. 284, R1269-R1276. doi: 10.1152/ajpregu.00442.2002

Hu, J. L., Nie, S. P., Min, F. F., and Xie, M. Y. (2012). Polysaccharide from seeds of Plantago asiatica L. increases short-chain fatty acid production and fecal moisture along with lowering $\mathrm{pH}$ in mouse colon. J. Agric. Food Chem. 60, 11525-11532. doi: 10.1021/jf302169u

Jiang, H., Ling, Z., Zhang, Y., Mao, H., Ma, Z., Yin, Y., et al. (2015). Altered fecal microbiota composition in patients with major depressive disorder. Brain Behav. Immun. 48, 186-194. doi: 10.1016/j.bbi.2015.03.016

Kelly, J. R., Kennedy, P. J., Cryan, J. F., Dinan, T. G., Clarke, G., and Hyland, N. P. (2015). Breaking down the barriers: the gut microbiome, intestinal permeability and stress-related psychiatric disorders. Front. Cell. Neurosci. 9:392. doi: 10.3389/fncel.2015.00392

Kiraly, D. D., Walker, D. M., Calipari, E. S., Labonte, B., Issler, O., Pena, C. J., et al. (2016). Alterations of the host microbiome affect behavioral responses to cocaine. Sci. Rep. 6:35455. doi: 10.1038/srep35455

Koh, A., De Vadder, F., Kovatcheva-Datchary, P., and Backhed, F. (2016). From dietary fiber to host physiology: short-chain fatty acids as key bacterial metabolites. Cell 165, 1332-1345. doi: 10.1016/j.cell.2016.05.041

Kunugi, H. (2016). Depressive disorder and gut-brain interaction. Brain Nerve 68, 641-646. doi: 10.11477/mf.1416200455 
Leclercq, S., Forsythe, P., and Bienenstock, J. (2016). Posttraumatic stress disorder: Does the gut microbiome hold the key? Can. J. Psychiatry 61, 204-213. doi: $10.1177 / 0706743716635535$

Lecomte, V., Kaakoush, N. O., Maloney, C. A., Raipuria, M., Huinao, K. D., Mitchell, H. M., et al. (2015). Changes in gut microbiota in rats fed a high fat diet correlate with obesity-associated metabolic parameters. PLOS ONE 10:e0126931. doi: 10.1371/journal.pone.0126931

Li, Q., and Zhou, J. M. (2016). The microbiota-gut-brain axis and its potential therapeutic role in autism spectrum disorder. Neuroscience 324, 131-139. doi: 10.1016/j.neuroscience.2016.03.013

Luna, R. A., and Foster, J. A. (2015). Gut brain axis: diet microbiota interactions and implications for modulation of anxiety and depression. Curr. Opin. Biotechnol. 32, 35-41. doi: 10.1016/j.copbio.2014.10.007

Macedo, D., Filho, A. J., Soares De Sousa, C. N., Quevedo, J., Barichello, T., Junior, H. V., et al. (2016). Antidepressants, antimicrobials or both? Gut microbiota dysbiosis in depression and possible implications of the antimicrobial effects of antidepressant drugs for antidepressant effectiveness. J. Affect. Disord. 208, 22-32. doi: 10.1016/j.jad.2016.09.012

MacFabe, D. F., Cain, D. P., Rodriguez-Capote, K., Franklin, A. E., Hoffman, J. E., Boon, F., et al. (2007). Neurobiological effects of intraventricular propionic acid in rats: possible role of short chain fatty acids on the pathogenesis and characteristics of autism spectrum disorders. Behav. Brain Res. 176, 149-169. doi: 10.1016/j.bbr.2006.07.025

MacFabe, D. F., Cain, N. E., Boon, F., Ossenkopp, K. P., and Cain, D. P. (2011). Effects of the enteric bacterial metabolic product propionic acid on object-directed behavior, social behavior, cognition, and neuroinflammation in adolescent rats: relevance to autism spectrum disorder. Behav. Brain Res. 217, 47-54. doi: 10.1016/j.bbr.2010.10.005

Million, M., Diallo, A., and Raoult, D. (2016). Gut microbiota and malnutrition. Microb. Pathog. 106, 127-138. doi: 10.1016/j.micpath.2016.02.003

Miyoshi, J., and Chang, E. B. (2016). The gut microbiota and inflammatory bowel diseases. Transl. Res. 179, 38-48. doi: 10.1016/j.trsl.2016.06.002

Moran, C. P., and Shanahan, F. (2014). Gut microbiota and obesity: role in aetiology and potential therapeutic target. Best Pract. Res. Clin. Gastroenterol. 28, 585-597. doi: 10.1016/j.bpg.2014.07.005

Neufeld, K. M., Kang, N., Bienenstock, J., and Foster, J. A. (2011). Reduced anxiety-like behavior and central neurochemical change in germ-free mice. Neurogastroenterol. Motil. 23, 255-264, e119. doi: 10.1111/j.1365-2982.2010. 01620.x

Parashar, A., and Udayabanu, M. (2016). Gut microbiota regulates key modulators of social behavior. Eur. Neuropsychopharmacol. 26, 78-91. doi: 10.1016/j. euroneuro.2015.11.002

Principi, N., and Esposito, S. (2016). Gut microbiota and central nervous system development. J. Infect. 73, 536-546. doi: 10.1016/j.jinf.2016.09.010

Rai, R., Saraswat, V. A., and Dhiman, R. K. (2015). Gut microbiota: its role in hepatic encephalopathy. J. Clin. Exp. Hepatol. 5, S29-S36. doi: 10.1016/j.jceh. 2014.12.003

Rea, K., Dinan, T. G., and Cryan, J. F. (2016). The microbiome: a key regulator of stress and neuroinflammation. Neurobiol. Stress 4, 23-33. doi: 10.1016/j.ynstr. 2016.03.001

Ridaura, V., and Belkaid, Y. (2015). Gut microbiota: the link to your second brain. Cell 161, 193-194. doi: 10.1016/j.cell.2015.03.033

Sarkar, A., Lehto, S. M., Harty, S., Dinan, T. G., Cryan, J. F., and Burnet, P. W. J. (2016). Psychobiotics and the manipulation of bacteria-gut-brain signals. Trends Neurosci. 39, 763-781. doi: 10.1016/j.tins.2016.09.002

Scheperjans, F., Aho, V., Pereira, P. A., Koskinen, K., Paulin, L., Pekkonen, E., et al. (2015). Gut microbiota are related to Parkinson's disease and clinical phenotype. Mov. Disord. 30, 350-358. doi: 10.1002/mds.26069

Severance, E. G., Yolken, R. H., and Eaton, W. W. (2016). Autoimmune diseases, gastrointestinal disorders and the microbiome in schizophrenia: more than a gut feeling. Schizophr. Res. 176, 23-35. doi: 10.1016/j.schres.2014.06.027
Shultz, S. R., Aziz, N. A., Yang, L., Sun, M., Macfabe, D. F., and O’brien, T. J. (2015). Intracerebroventricular injection of propionic acid, an enteric metabolite implicated in autism, induces social abnormalities that do not differ between seizure-prone (FAST) and seizure-resistant (SLOW) rats. Behav. Brain Res. 278, 542-548. doi: 10.1016/j.bbr.2014.10.050

Sinha, R., and Jastreboff, A. M. (2013). Stress as a common risk factor for obesity and addiction. Biol. Psychiatry 73, 827-835. doi: 10.1016/j.biopsych.2013.01.032

Skosnik, P. D., and Cortes-Briones, J. A. (2016). Targeting the ecology within: the role of the gut-brain axis and human microbiota in drug addiction. Med. Hypotheses 93, 77-80. doi: 10.1016/j.mehy.2016.05.021

Song, X., Xu, Z., Liu, Q., Li, Y., Ma, Y., Wang, J., et al. (2016). Comparative study of the composition and genetic diversity of the picoeukaryote community in a Chinese aquaculture area and an open sea area. J. Mar. Biol. Assoc. U.K. 97, 151-159. doi: 10.1017/S0025315416000205

Stilling, R. M., Van De Wouw, M., Clarke, G., Stanton, C., Dinan, T. G., and Cryan, J. F. (2016). The neuropharmacology of butyrate: the bread and butter of the microbiota-gut-brain axis? Neurochem. Int. 99, 110-132. doi: 10.1016/j.neuint. 2016.06.011

Sudo, N. (2016). Effects of gut microbiota on stress response and behavioral phenotype of the host. Brain Nerve 68, 595-605. doi: 10.11477/mf.1416200447

Tarr, A. J., Galley, J. D., Fisher, S. E., Chichlowski, M., Berg, B. M., and Bailey, M. T. (2015). The prebiotics 3'Sialyllactose and 6'Sialyllactose diminish stressorinduced anxiety-like behavior and colonic microbiota alterations: evidence for effects on the gut-brain axis. Brain Behav. Immun. 50, 166-177. doi: 10.1016/j. bbi.2015.06.025

Tillisch, K., Labus, J., Kilpatrick, L., Jiang, Z., Stains, J., Ebrat, B., et al. (2013). Consumption of fermented milk product with probiotic modulates brain activity. Gastroenterology 144, 1394-1401, 1401.e1-e4. doi: 10.1053/j.gastro. 2013.02.043

Volpe, G. E., Ward, H., Mwamburi, M., Dinh, D., Bhalchandra, S., Wanke, C., et al. (2014). Associations of cocaine use and HIV infection with the intestinal microbiota, microbial translocation, and inflammation. J. Stud. Alcohol Drugs 75, 347-357. doi: 10.15288/jsad.2014.75.347

Watanabe, Y., Nagai, F., and Morotomi, M. (2012). Characterization of Phascolarctobacterium succinatutens sp. nov., an asaccharolytic, succinateutilizing bacterium isolated from human feces. Appl. Environ. Microbiol. 78, 511-518. doi: 10.1128/AEM.06035-11

Wu, H., Tremaroli, V., and Backhed, F. (2015). Linking microbiota to human diseases: a systems biology perspective. Trends Endocrinol. Metab. 26, 758-770. doi: $10.1016 /$ j.tem.2015.09.011

Xue, Y. X., Luo, Y. X., Wu, P., Shi, H. S., Xue, L. F., Chen, C., et al. (2012). A memory retrieval-extinction procedure to prevent drug craving and relapse. Science 336, 241-245. doi: 10.1126/science. 1215070

Yang, J., Martinez, I., Walter, J., Keshavarzian, A., and Rose, D. J. (2013). In vitro characterization of the impact of selected dietary fibers on fecal microbiota composition and short chain fatty acid production. Anaerobe 23, 74-81. doi: 10.1016/j.anaerobe.2013.06.012

Yano, J. M., Yu, K., Donaldson, G. P., Shastri, G. G., Ann, P., Ma, L., et al. (2015). Indigenous bacteria from the gut microbiota regulate host serotonin biosynthesis. Cell 161, 264-276. doi: 10.1016/j.cell.2015.02.047

Conflict of Interest Statement: The authors declare that the research was conducted in the absence of any commercial or financial relationships that could be construed as a potential conflict of interest.

Copyright $(2017$ Ning, Gong, Xie and Ma. This is an open-access article distributed under the terms of the Creative Commons Attribution License (CC BY). The use, distribution or reproduction in other forums is permitted, provided the original author(s) or licensor are credited and that the original publication in this journal is cited, in accordance with accepted academic practice. No use, distribution or reproduction is permitted which does not comply with these terms. 